\title{
An Electronic Patch for Wearable Health Monitoring by Reflectance Pulse Oximetry
}

Haahr, Rasmus Grønbek; Duun, Sune Bro; Toft, Mette H.; Belhage, Bo; Larsen, Jan; Birkelund, Karen; Thomsen, Erik Vilain

Published in:

I E E E Transactions on Biomedical Circuits and Systems

Link to article, DOI:

10.1109/TBCAS.2011.2164247

Publication date:

2012

Link back to DTU Orbit

Citation (APA):

Haahr, R. G., Duun, S. B., Toft, M. H., Belhage, B., Larsen, J., Birkelund, K., \& Thomsen, E. V. (2012). An Electronic Patch for Wearable Health Monitoring by Reflectance Pulse Oximetry. I E E E Transactions on Biomedical Circuits and Systems, 6(1), 45-53. https://doi.org/10.1109/TBCAS.2011.2164247

\section{General rights}

Copyright and moral rights for the publications made accessible in the public portal are retained by the authors and/or other copyright owners and it is a condition of accessing publications that users recognise and abide by the legal requirements associated with these rights.

- Users may download and print one copy of any publication from the public portal for the purpose of private study or research.

- You may not further distribute the material or use it for any profit-making activity or commercial gain

- You may freely distribute the URL identifying the publication in the public portal 


\title{
An Electronic Patch for Wearable Health Monitoring by Reflectance Pulse Oximetry
}

\author{
Rasmus G. Haahr, Member, IEEE, Sune B. Duun, Mette H. Toft, Bo Belhage, Jan Larsen, Senior Member, IEEE, \\ Karen Birkelund, and Erik V. Thomsen
}

\begin{abstract}
We report the development of an Electronic Patch for wearable health monitoring. The Electronic Patch is a new health monitoring system incorporating biomedical sensors, microelectronics, radio frequency ( $R F)$ communication, and a battery embedded in a 3-dimensional hydrocolloid polymer. In this paper the Electronic Patch is demonstrated with a new optical biomedical sensor for reflectance pulse oximetry so that the Electronic Patch in this case can measure the pulse and the oxygen saturation. The reflectance pulse oximetry solution is based on a recently developed annular backside silicon photodiode to enable low power consumption by the light emitting components. The Electronic Patch has a disposable part of soft adhesive hydrocolloid polymer and a reusable part of hard polylaurinlactam. The disposable part contains the battery. The reusable part contains the reflectance pulse oximetry sensor and microelectronics. The reusable part is 'clicked' into the disposable part when the patch is prepared for use. The patch has a size of $88 \mathrm{~mm}$ by $60 \mathrm{~mm}$ and a thickness of $5 \mathrm{~mm}$.
\end{abstract}

Index Terms-Blood oxygen saturation, Electronic Patch, photoplethysmography (PPG), reflectance pulse oximetry, wearable health monitoring.

\section{INTRODUCTION}

D EVELOPMENT of wearable health systems for physiological monitoring have made substantial progress within the last years. During the last decades, state-of-the-art have been bulky systems with boxes strapped onto the body and connected by wires to biomedical sensors, e.g., the Holter monitors [1]. Recent advancements in microelectronics and radio communication have increased the computational power, decreased the form factor and power consumption of microprocessors. This has enabled the development of wearable monitoring systems where sensors, advanced microcontroller units, digital signal processing, and radio frequency communication are built into

Manuscript received December 02, 2010; revised April 21, 2011; accepted July 14, 2011. This paper was recommended by Associate Editor Emil Jovanov.

R. G. Haahr is with the Department of Micro and Nanotechnology, Technical University of Denmark, 2800 Kgs. Lyngby, Denmark and also with DELTA Microelectronics, Venlighedsvej 4, 2970 Hørsholm, Denmark (e-mail: rashaahr@gmail.com).

S. B. Duun, K. Birkelund, and E. V. Thomsen are with the Department of Micro and Nanotechnology, Technical University of Denmark, 2800 Kgs. Lyngby, Denmark (e-mail: karen.birkelund@nanotech.dtu.dk; erik.v.thomsen@nanotech.dtu.dk).

M. H. Toft and B. Belhage are with the Bispebjerg Hospital, University of Copenhagen, 2400 Copenhagen NV, Denmark.

J. Larsen is with the Department of Informatics and Mathematical Modeling, Technical University of Denmark, 2800 Kgs. Lyngby, Denmark.

Color versions of one or more of the figures in this paper are available online at http://ieeexplore.ieee.org.

Digital Object Identifier 10.1109/TBCAS.2011.2164247

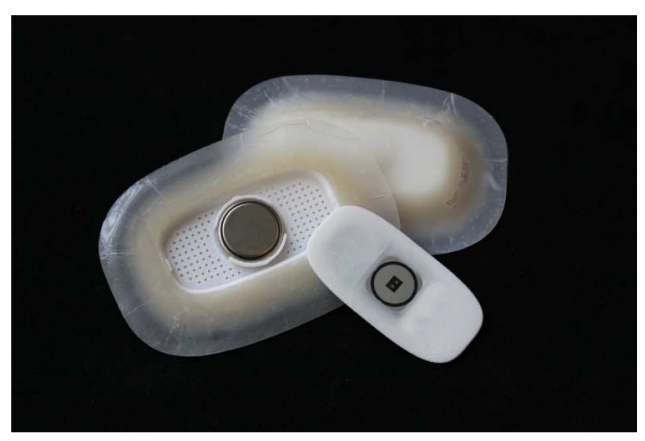

Fig. 1. The picture shows the Electronic Patch with the developed photoplethysmographic sensor. The sensor consist of commercial light emitting diodes and a specially designed annular photodiode. Besides the photoplethysmographic sensor the Electronic Patch also contains electronics for signal processing, wireless radio communication and a coin cell battery. These components are embedded in an hydrocolloid adhesive material. The patch has a size of $88 \mathrm{~mm}$ by $60 \mathrm{~mm}$ and is $5 \mathrm{~mm}$ thick.

devices that can be worn conveniently and discreetly. These devices rely on a new infrastructure in the healthcare system which integrates information technology (i.e., telemedicine) in contrast to traditional physician examination.

Telemedicine technologies were first developed as professional physician-to-physician systems, e.g., in treatment and managing of an acute stroke a system, known as "Telestroke", has been described in 1999 [2] and proven to benefit treatment of patients [3]. Likewise, telemedicine has been introduced to patients as home-based installations. This has successfully been applied to patients with chronic heart conditions [4]-[6].

Wearable telemedicine solutions are emerging and a number of approaches for wearable monitoring systems have been studied:

Systems worn around the wrist have been developed by J. M. Kang et al. [7] and U. Anliker et al. [8]. A wireless reflectance pulse oximeter for the forehead has been described by Y. Mendelson et al. [9]. Clothes and textiles with built-in sensors and compatible with supporting wireless devices have been investigated by S. Park et al. [10] and R. Paradiso et al. [11], [12].

The Electronic Patch (Fig. 1) presented in this paper advances the field of wearable health monitoring devices by introducing a new method and technology to embed sensors, power source, and data handling in an adhesive material which unites to the body. In contrast to the above mentioned technologies and alike, the Electronic Patch is a single unit without wires and it does not limit movement. It can be worn at all times including, e.g., a shower. These features are essential for long-term monitoring. 


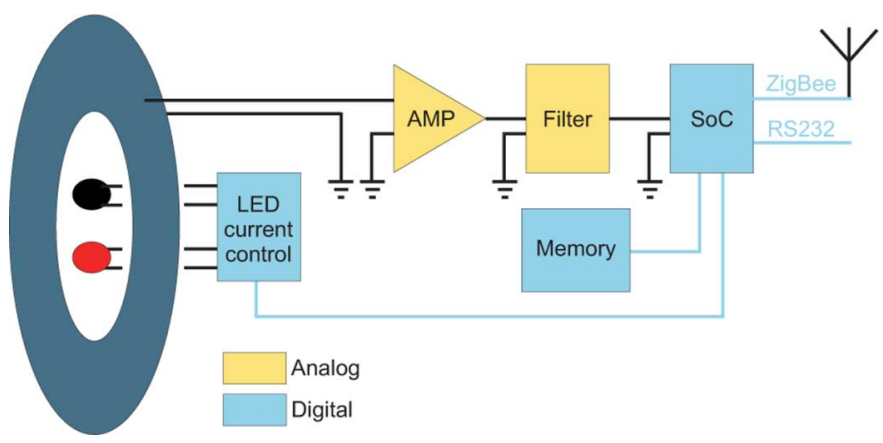

Fig. 2. The figure illustrates the architecture of the system. The annular photodiode is seen to the left with the two LEDs. The analog amplifiers and filters use low-power operational amplifiers. The SoC is seen to the right, a CC2430 (Texas Instruments) with integrated $2.4 \mathrm{GHz}$ wireless communication. The system has 64 kbit EEPROM memory. The LED current controller is a MAX6947 (Maxim Integrated Products).

We have previously presented the vision of the Electronic Patch [13], development of a new pulse oximetry sensor based on an annular photodiode [14], [15] and preliminary work on the packaging of the sensor and electronics, and mechanical assembly of the Electronic Patch [16].

In this paper, the technology of the Electronic Patch is described in detail with emphasis on the encapsulation of the sensor and electronics embedded in the adhesive material. The paper includes description of the firmware, data acquisition, and a preliminary clinical evaluation against a standard pulse oximeter. The scope of this paper is to present a device for new healthcare applications and management of chronic diseases rather than optimized electronics and firmware for wearable health monitoring devices as reported by Yazicioglu et al. [17], Wong et al. [18], and Tavakoli et al. [19]. We have presented aspects of the device including the annular photodiode in previous work [14]-[16].

Pulse oximetry is a non-invasive optical method to obtain both physiological (i.e., pulse rate) and biochemical information $\left(\mathrm{SpO}_{2}\right)$. This fact makes it very desirable to employ in wearable sensor systems. Pulse oximetry was invented in 1972 by T. Aoyagi [20]. Reflectance pulse oximetry was first demonstrated by Y. Mendelson in 1983 [21]. The two wavelengths employed are typically red $(660 \mathrm{~nm})$ and infrared $(940 \mathrm{~nm})$ because of the characteristics of the absorption spectra of deoxygenated hemoglobin $(\mathrm{Hb})$ and oxygenated hemoglobin $(\mathrm{HbO})$ [14]. A challenge with pulse oximetry in relation to wearable health monitoring devices is the higher power consumption compared to electrical measurements, e.g., ECG, due to the light sources. To reduce the power consumption we have developed a lowpower pulse oximetry sensor which is described elsewhere [15].

\section{EleCtronic PATCh System}

The Electronic Patch is made for pulse oximetry. Other types of biomedical sensors could however be implemented to the Electronic Patch. The patch is intended for use on the upper part of the truncus where it is conveniently located behind clothes.

The architecture of the Electronic Patch is seen in Fig. 2 and a conceptual illustration in Fig. 3. The Electronic Patch contains a central printed circuit board (PCB) with a recently developed 2D annular photodiode [15] to decrease the power consumption

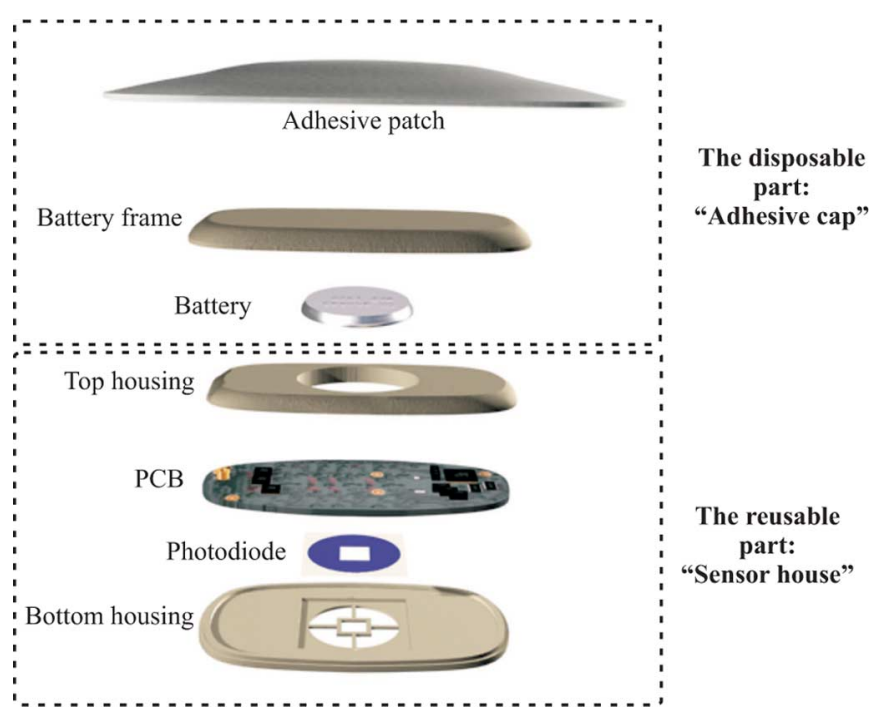

Fig. 3. The figure illustrates the mechanical assembly of the Electronic Patch. The upper, disposable, part contains the adhesive and the battery attached in the top of the battery frame. This part is designated "adhesive cap". The lower part is reusable and contains the electronics and reflectance pulse oximetry sensor encapsulated in a hard plastic housing. This part is designated "sensor house".

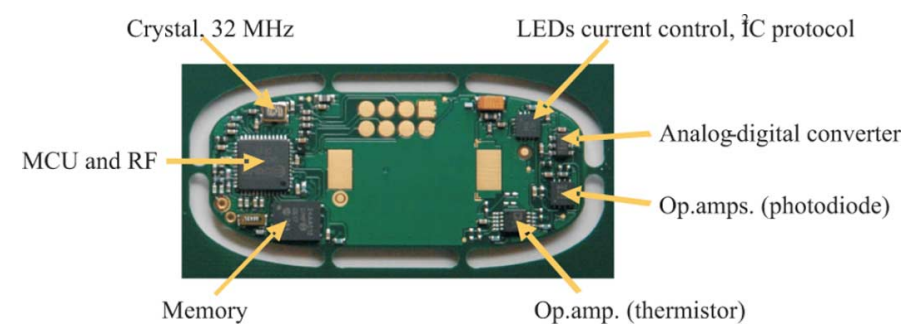

Fig. 4. The top side of the PCB showing the layout of the electronic components. The space in the middle is reserved for the battery.

by the light emitting diodes (LEDs). The PCB is then encapsulated in first a hard plastic housing and then in a soft adhesive material.

The scope of the Electronic Patch is to demonstrate the concept of a patch device with integrated pulse oximetry. We have employed state-of-the-art (as of primo 2007) commercial components. Since the focus of this paper is the method and technology of the Electronic Patch the electronics and firmware is developed as a baseline solution with limited focus on power optimization.

In Sections III-V each individual part and procedure of the system is described.

\section{A. Electronics}

The central printed circuit board is seen in Fig. 4. The main active components are a CC2430 (Texas Instruments) system-on-chip (SoC) with $2.4 \mathrm{GHz}$ wireless communication, 64 kbit electrically erasable programmable read-only memory (EEPROM), and a MAX6947 (Maxim Integrated Products) current controller. The right hand side of the PCB contains the analog parts and the left hand side contains the digital parts. The signal from the photodiode is amplified by first a transimpedance amplifier; an operational amplifier in inverting configuration with a feedback resistor of $20 \mathrm{k} \Omega$ and capacitor 


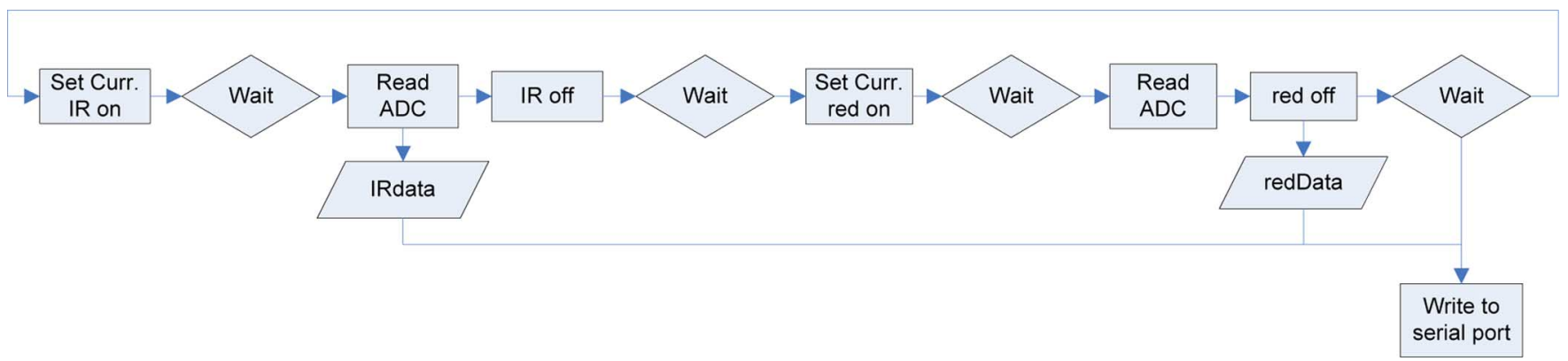

Fig. 5. The figure illustrates the architecture of the microcontroller's sampling algorithm. The algorithm has the following sequence: Measure the background level, turn the IR LED on, wait to avoid transients, measure, and turn off the IR LED. When the red LED is turned on a similar sampling process is repeated. Each cycle returns two values, one with the IR LED on and one with the red LED on. These values are then sent to the serial port or radio.

of $1 \mathrm{nF}$. Second, a voltage amplifier; an operational amplifier in non-inverting configuration and with a $200 \Omega$ both as feedback capacitor and resistance to ground. The feedback capacitor is $100 \mathrm{pF}$. The CC2430 has a built-in 12 bits analog-to-digital converter (ADC). Two crystals support the CC2430, one at $32 \mathrm{kHz}$ and another at $32 \mathrm{MHz}$. The CC2430, MAX6947, and memory communicates over the $\mathrm{I}^{2} \mathrm{C}$ bus at $400 \mathrm{kHz}$ with 7 bits addressing. Two types of data communication interfaces are supported: 1) A wired using the RS232 serial interface and 2) a wireless using the $2.4 \mathrm{GHz}$ networking. In this study the RS232 serial interface was used since there was no need for wireless operation to evaluate the sensor.

The electronic components, apart from the photodiode, are soldered to the PCB using standard surface mounting technology. The photodiode is mounted using a CW2400 conducting epoxy (Circuitworks) and a Chipcoat 8426 underfiller (Namics) for good mechanical adhesion.

\section{B. Firmware}

The patch runs an algorithm for collection and transmission of data. The architecture of the algorithm is illustrated in Fig. 5. The overall principle is that the patch records data and transmits it for further evaluation. In a study by our collaborators the data transmission from the patient at home to a hospital have been demonstrated, the results still remain to be published. The patch can transmit using either the RS232 standard or ZigBee. To evaluate the integration of the reflectance pulse oximetry the RS232 port has been employed.

The firmware has two tasks; 1) record data 2) send data. The first task requires precise timing and the second task can be performed whenever resources are available. For the first task the MCU's timer is used, this timed sequence is seen in Fig. 6. The graph shows two sampling cycles (corresponding to the two groups of dual peaks) of the photocurrent generated by the red and the IR LEDs. The green curve is a logic condition representing the periods of reading the analog to digital converter. The black curve is the analog output from the photodiode after amplification. When this signal is high one of the LEDs is turned on. The IR LED has a higher output compared to the red signal when measuring light returning from the tissue. This is primarily due to a difference in quantum efficiency of the photo detector, and secondly due to differences in absorbance of the two wavelengths in tissue and differences in quantum efficiency of the LEDs. To avoid turn-on transients in both the LED and the am-

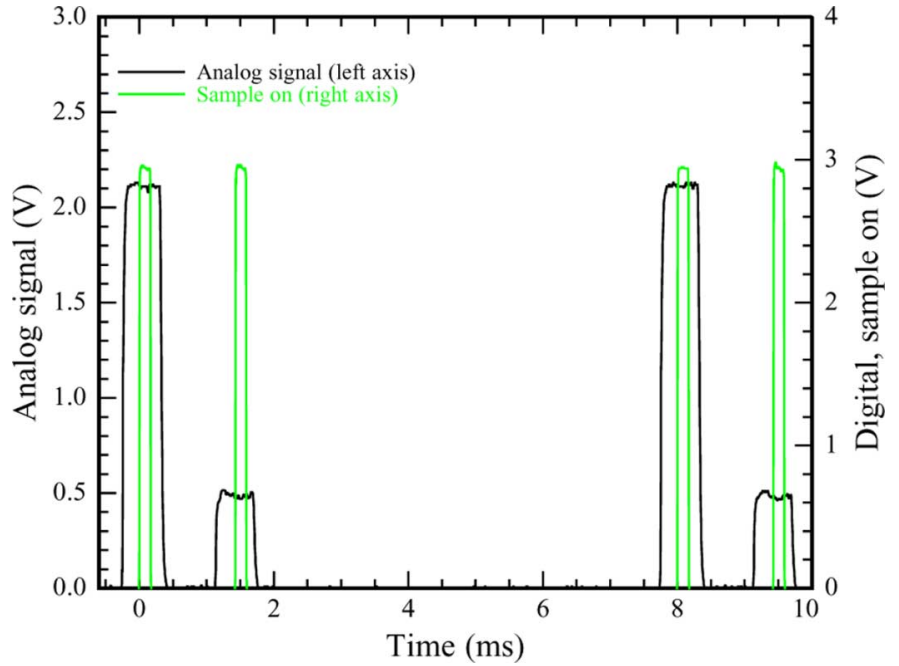

Fig. 6. The graph shows the timing and duty cycle of the LEDs. The green color is a digital signal showing the periods where the ADC is active. The black color is the analog output from the photodiode after amplification. The graph shows two groups of dual peaks. Each represent a cycle with the IR LED turned on followed by the red LED turned on. The sampling rate is seen to be $125 \mathrm{~Hz}$ with a duty cycle of $14 \%$

plifying circuity, the LED is turned on $275 \mu$ s before initiating sampling. The duty cycle of the LED is $14 \%$ when sampling at $125 \mathrm{~Hz}$ as seen in Fig. 6.

As data is sampled, it is sent to the universal asynchronous receiver/transmitter (UART) in ASCII containing the following information: Sequence number, IR measurement value, and red measurement value. The media access control (MAC) layer in the SoC transmits the data over the RS232 interface whenever resources are not used by the sampling task.

\section{Sensor}

The sensor comprises two commercial LEDs, at wavelengths of $660 \mathrm{~nm}$ (Lumex Inc.) and $940 \mathrm{~nm}$ (Stanley Electric Co., Ltd.), placed in the center of an annular backside silicon photodiode. The concept of this geometry was first presented by Haahr $e t$ $a l$. [13]. The annular photodiode is used to reduce the current consumption in the LEDs as proposed by Mendelson et al. [22], [23] by usage of several discrete components. The annular photodiode is prepared in several geometric configurations with different inner and outer radii. The different geometries of the photodiodes are listed in Table I. Each geometry is referred to as a 
TABLE I

DimENSIONS OF THE ANNULAR PHOTODIODES

\begin{tabular}{|l|r|r|r|}
\hline Type & $\begin{array}{r}\text { Inner radius } \\
{[\mathrm{mm}]}\end{array}$ & $\begin{array}{r}\text { Outer radius } \\
{[\mathrm{mm}]}\end{array}$ & $\begin{array}{r}\text { Area } \\
{\left[\mathrm{mm}^{2}\right]}\end{array}$ \\
\hline $\mathrm{B}$ & 3 & 4 & 22 \\
\hline $\mathrm{C}$ & 4 & 5 & 28 \\
\hline $\mathrm{D}$ & 5 & 6 & 35 \\
\hline $\mathrm{E}$ & 6 & 7 & 40 \\
\hline $\mathrm{F}$ & 3 & 7 & 117 \\
\hline
\end{tabular}

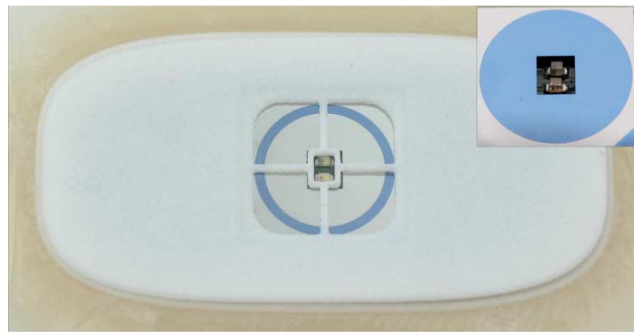

Fig. 7. The photo shows an E-type photodiode packaged and assembled into a patch. The two LEDs are seen in the center. The chip in the upper right corner shows a F-type photodiode with a micro-structured surfaced for better absorption of light.

type, e.g., an F-type photodiode has inner radius of $3 \mathrm{~mm}$ and outer radius of $7 \mathrm{~mm}$. Fig. 7 shows an E-type annular photodiode embedded in the Electronic Patch with LEDs seen in the middle; an F-type photodiode is shown in the top right corner of the figure.

Backside photodiodes are chosen because all contacts are on the backside and hence there is no need for wirebonding, which makes the assembly and packaging easier.

Some of the photodiodes have a micro-structured surface for better absorption at all angles of incidence. The micro-structuring is done by a potassium hydroxide $(\mathrm{KOH})$ etch. A surface-structured photodiode is seen in the upper right corner of Fig. 7.

To ensure that only light from the desired distance away from the LEDs is detected, the aperture of the photodiode is defined on the surface of the photodiode with an aluminium layer. The aluminium layer is seen as the white parts of the photodiodes in Fig. 7.

The quantum efficiency for one of the E-type photodiodes is shown in Fig. 8. The quantum efficiency is seen to have a maximum of $60 \%$. A detailed description of the fabrication and characterization of the photodiodes are described in [15], [24].

\section{Energy Supply and Power Consumption}

The Electronic Patch is powered by a CR2025 coin size battery, delivering $3 \mathrm{~V}$ and $170 \mathrm{mAh}$.

The total current consumption of the sensors and electronics when supplied with $3.0 \mathrm{~V}$ is measured using an Agilent E3611A. The results are shown in Table II. The baseline firmware solution does not perform power regulation tasks. The $\mathrm{SoC}$ is therefore constantly active, which gives a high current consumption. The current consumption is $18.2 \mathrm{~mA}$ during measurement with data transmission over the RS232 port, LEDs on, and the SoC active. The power is primarily used by the SoC and radio as the LEDs only use an average of $2.8 \mathrm{~mA}$. It is also seen from Table II that

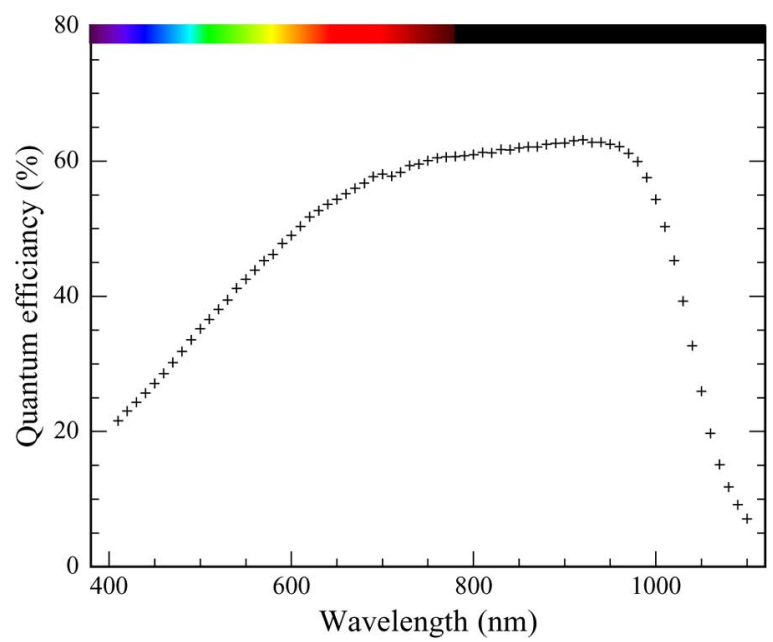

Fig. 8. The figure shows the quantum efficiency for one of the E-type photodiodes. The quantum efficiency is seen to have a maximum of $60 \%$.

TABLE II

CURRENT CONSUMPTION OF ElECTRONICS AND SENSORS WHEN SUPPLIED WITH 3.0 V. THE DUTY CYCLE OF THE LEDS IS $14 \%$

\begin{tabular}{|l|r|}
\hline SoC idle & $0.2 \mathrm{~mA}$ \\
\hline SoC active, LEDs off & $12.4 \mathrm{~mA}$ \\
\hline SoC active, LEDs @ 10mA \& 200 Hz & $15.2 \mathrm{~mA}$ \\
\hline $\begin{array}{l}\text { SoC active, LEDs @ 10mA \& 200 Hz } \\
\text { and sending on serial line (wire) }\end{array}$ & $18.2 \mathrm{~mA}$ \\
\hline $\begin{array}{l}\text { SoC active, LEDs @, 10mA \& } 200 \mathrm{~Hz} \\
\text { and sending on radio }\end{array}$ & $33 \mathrm{~mA}$ \\
\hline
\end{tabular}

the SoC has an idle mode with a current consumption of $0.2 \mathrm{~mA}$.

In wireless measurements the current consumption is $33 \mathrm{~mA}$. To avoid draining the battery the data cannot continuously be streamed over the radio. However, it is possible to send an alarm upon detection of an event, e.g., the oxygen saturation fall below a certain threshold.

\section{E. Packaging}

The Electronic Patch comes in two parts, a reusable and a disposable: The reusable part, the sensor housing, contains the sensors and electronics encapsulated in a plastic housing as seen in the lower part of Fig. 3. The disposable part, the adhesive cap, comprise the battery frame and battery embedded in an adhesive patch as seen in the upper part of Fig. 3. The Electronic Patch is assembled by 'clicking' the sensor house into the adhesive cap. The two parts are held together with snap latches. The two part construction is chosen so that the expensive parts (sensor and electronics) can be reused while the adhesive cap is disposable.

The sensor house has the dimensions $56 \mathrm{~mm} \times 28 \mathrm{~mm}$ and is $4 \mathrm{~mm}$ thick. The adhesive cap has dimensions of $88 \mathrm{~mm} \times$ $60 \mathrm{~mm}$ and is $5 \mathrm{~mm}$ thick. This is also the dimensions of the assembled patch. The weight of the assembled patch is $16 \mathrm{~g}$.

The plastic parts (bottom housing, top housing and battery frame) are currently manufactured in polylaurinlactam (PA12 or Nylon) using Selective Laser Sintering (SLS) a 3D printing technique which is an easy and low cost method for rapid prototyping. The used PA12 is USP class VI approved i.e., bio-compatible. Adhesive (Loctite 4031) is used for assembly of the PCB in the housing and the battery in the battery frame. 
TABLE III

The Composition of the AdHesive Cap, Disposable Part, FROM TOP TO BOTTOM

\begin{tabular}{|l|r|}
\hline Function & Material/Chemical name \\
\hline Backing & Plastic film \\
\hline Adhesive & Adhesive containing hydrocollorid polymer \\
\hline Plastic capes & Polylaurinlactam \\
\hline Battery & CR2025 \\
\hline Release liner & Polyethylene terephthalate (PET) \\
\hline Non-touch & Polyester \\
\hline
\end{tabular}

The adhesive material is a mixture of a water-swellable hydrocolloid and a water-insoluble, viscous, and elastomeric binder [25] (Coloplast A/S). It is shaped to fit the adhesive cap of the Electronic Patch. The composition of the adhesive cap is seen in Table III. Adhesive caps with protective films removed are shown in Fig. 1.

The Electronic Patch has been tested for skin irritation at Coloplast A/S: Patches have been worn for a week by test persons during normal daily activities including sports and bathing without detaching. It has also been tested by Coloplast $\mathrm{A} / \mathrm{S}$ that the patches do not pose any toxicological concern for the proposed usages, if used by persons with a body weight above $10 \mathrm{~kg}$.

The hole in the bottom housing is covered with an optically transparent and bio-compatible epoxy Epo-Tek 302-3M (Epoxy Technology Inc.) to protect the photodiode chip. The epoxy has a thickness of approximately $300 \mu \mathrm{m}$. The refractive index of the epoxy is 1.56 which is close to the refractive index of the human skin. (In human skin the refractive index of the outer skin layer, the epidermis, is approximately 1.43 at $660 \mathrm{~nm}$ and 1.42 at $940 \mathrm{~nm}[26])$.

The optical filter of the photodiode is designed to the epoxy interface. Hence, it is matched to the refractive index 1.56 of the epoxy. The photodiode has a one layer silicon nitride $\left(\mathrm{Si}_{x} \mathrm{~N}_{y}\right)$ on silicon interference filter to optimize transmission. The silicon nitride has a thickness of $93 \mathrm{~nm}$ and a refractive index of 2.09. It is important that the epoxy has an optical thickness greater than the typical coherence length of the LEDs to avoid unwanted interference. The coherence length of an typical LED is 50-100 $\mu \mathrm{m}$ and the optical thickness of the epoxy layer is approximately $470 \mu \mathrm{m}$.

The transmission is better than $90 \%$ at wavelengths $660 \mathrm{~nm}$ and $940 \mathrm{~nm}$ at angles of incidence ranging from $0^{\circ}$ to $60^{\circ}$.

\section{Clinical Evaluation}

The sensor of the Electronic Patch has been clinically evaluated with respect to measurement of 1) pulse and 2) $\mathrm{SpO}_{2}$ against a reference pulse oximeter. The scope has been to test the sensor in an controlled environment in a standard setup. Results relating to the user model of the Electronic Patch is not covered as part of evaluating the sensor.

\section{A. Method and Materials}

The evaluation of the sensor is performed as a self-testing study using three subjects. ${ }^{1}$ The experiment is done by lowering the oxygen saturation from $100 \%$ to approximately $75 \%$ over a

\footnotetext{
${ }^{1}$ The experiment is performed in accordance with the Helsinki Declaration and Danish law.
}

period of approximately $15 \mathrm{~min}$. The final saturation and duration of decreased oxygen saturation vary from subject to subject. To lower the oxygen saturation the subjects re-breath atmospheric air from a closed system of 50-70 liters. Carbon dioxide $\left(\mathrm{CO}_{2}\right)$ is absorbed from the circuit by breathing through a filter of calcium hydroxide, Soda Lime (Dräger). At the beginning of each experiment the subjects take a few inspirations of a $100 \%$ oxygen before switching to the closed circuit.

The reference oxygen saturation is measured with a commercial Datex-Ohmeda AS/3 Compact Patient Monitor, Pulse Oximeter. The Electronic Patch is placed on the third digit of the left hand as this is a location similar to the reference pulse oximeter which has a conventional sensor finger probe. This avoids problems with different desaturation rates at different monitoring sites. An E-type photodiode is used for the experiment as a pre-experiment screening showed that this geometry measured larger amplitudes of the PPG signals. The patch is connected to a computer by the RS232 serial interface. A LabView program collects the raw data. The Electronic Patch sends two raw PPG signals, one for the red and one for the IR signal, over the serial port. The data is recorded continuously and split into sections of $30 \mathrm{~s}$. The reference $\mathrm{SpO}_{2}$ is read off at the beginning and ending of each section.

\section{B. Data Analysis}

Following data acquisition of all subjects the following digital signal processing is performed to the measured data: 1) Normalization with respect to the average and 2) bandpass filtering using an 8th order Butterworth filter with $0.8 \mathrm{~Hz}$ and $3 \mathrm{~Hz}$ as the lower and higher cutoff frequencies. The cutoff frequencies are found by minimizing the mean square error of the $\mathrm{SpO}_{2}$ in a leave-one-out evaluation scheme as described below. The most suitable cutoff frequencies will depend on the pulse rate. Therefore, the cutoff frequencies should be adjusted for new data sets based on the criteria of minimizing the leave-one-out mean square error in the estimation of the $\mathrm{SpO}_{2}$. However, this step is only necessary in the process of calibrating and evaluating the sensor. A dynamic implementation could be made by changing the cutoff frequencies based on the estimation of the pulse rate. For this experiment the subjects are at rest with a pulse rate of approximately 60 beats per minute.

To estimate the $\mathrm{SpO}_{2}$ Mean Field Independent Component Analysis (ICAMF) is used. In a previous study, we have shown that ICAMF is a very suitable algorithm to use in pulse oximetry and has superior performance to other ICA approaches and the Masimo DST [27]. In this implementation the ICAMF is defined as

$$
\begin{aligned}
x_{r}(t) & =a_{r} s(t)+e_{r}(t) \\
x_{i r}(t) & =a_{i r} s(t)+e_{i r}(t)
\end{aligned}
$$

where $x_{r}(t)$ and $x_{i r}(t)$ are the measured signals, $s(t)$ is the PPG signal, $a_{r}$ and $a_{i r}$ are the mixing coefficients scaling the true PPG signal, and $e_{r}$ and $e_{i r}$ are noise terms. The ratio of the two mixing coefficients is defined as the optical ratio, $R$,

$$
R=\frac{a_{r}}{a_{i r}} \text {. }
$$



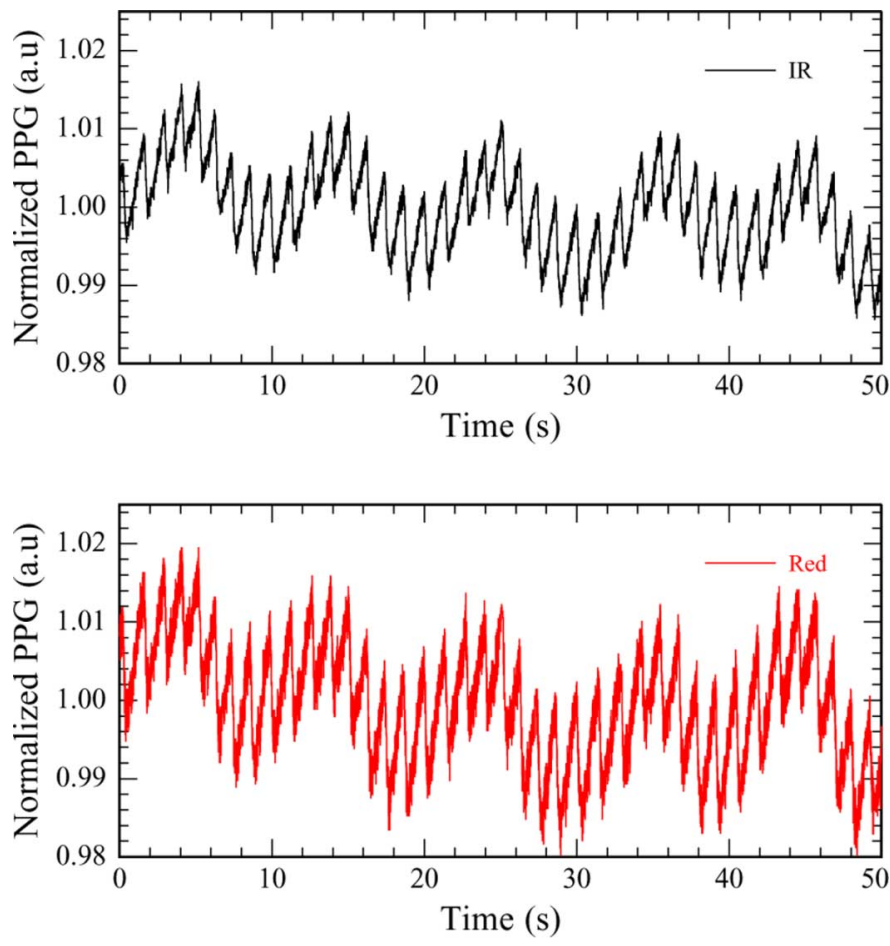

Fig. 9. The two plots show a 50 s sequence of the infrared and red PPGs at a saturation of $83 \%$ read off from Datex pulse oximeter. The PPGs are measured at the third digit on the left hand. Two frequency components are seen: One with a period of $1.3 \mathrm{~s}$ which relates to the heart beat, and one with a period of $10 \mathrm{~s}$ which relates to the respiration. The waveforms are sampled at $125 \mathrm{~Hz}$.

This ratio is thus a function of the oxygen saturation. Since $R$ should be positive, $a_{r}$ and $a_{i r}$ are constrained to positive values. We have applied a bi-Gaussian distribution in the source term and a diagonal covariance matrix for the noise terms as described in [27].

A leave-one-out training and test scheme has been applied to the data set to first estimate the calibration curve and second test the calibration. The data set is made up of three subjects and the scheme is repeated for all combinations. The final calibration curve is computed as an average over the three combinations. The final performance is the average of the mean square errors of the test set predictions over the three combinations.

\section{Results}

Fig. 9 shows a 50 s section of the recorded IR and red PPGs. ${ }^{2}$ The sections are recorded at a saturation level of $83 \%$ measured by the Datex pulse oximeter. Both the pulse and respiration are clearly seen with a period of $1.3 \mathrm{~s}$ and $10 \mathrm{~s}$, respectively.

The result of the data analysis is seen in Fig. 10 as the measured $\mathrm{SpO}_{2}$ values against the optical ratio, $R$. It is seen that there is a quasi linear relation [28] between $\mathrm{SpO}_{2}$ and $R$ in the $\mathrm{SpO}_{2}$ range $100 \%$ to $80 \%$. The average mean square error is found to be, in $\mathrm{SpO}_{2} \%, 2.6 \%$.

\footnotetext{
${ }^{2}$ The PPGs are plotted as recorded from the photo detector. Commercial pulse oximeters flip the $y$-axis so that the PPGs follow the plethysmogram i.e., systole at maximum and diastole at minimum.
}

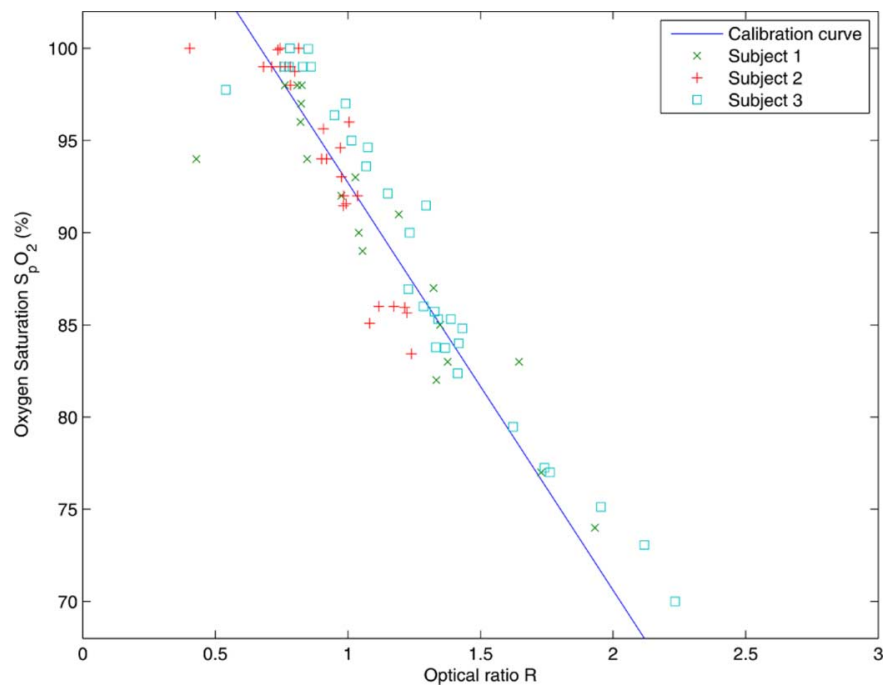

Fig. 10. The graph shows the calculated $\mathrm{SpO}_{2}$ values obtained from three subjects. Mean field independent components analysis has been used to calculate the optical ratio $R$. A calibration curve for the reflectance pulse oximeter is then found as shown in the graph [27].

\section{DISCUSSION}

The purpose of this work is to demonstrate the development and integration of a reflectance pulse oximetry solution into an Electronic Patch. In the following we discuss our solution and provide suggestions for improvements.

\section{A. Clinical Results}

The clinical evaluation demonstrated that the pulse and $\mathrm{SpO}_{2}$ can be measured equivalent to a standard pulse oximeter. In Fig. 9, the respiration is more clearly evident than usually seen in PPGs recorded at the finger. During the clinical experiments, we have noticed that the respiration is seen more clearly when the person is breathing through the mask and tube in the closed system. We believe the resistance in the system causes a larger pressure difference intrathoracic between inspiration and expiration to compensate and sustain a constant tidal volume. This will be evident in the arterial blood pressure and therefore also in the PPG.

This study was limited to comparing the sensor in the Electronic Patch against a standard pulse oximeter. The Electronic Patch was applied at the finger instead of a site more suitable for the Electronic Patch such as the chest to avoid a problem with a different desaturation rate compared to the desaturation rate at the finger. A typical pulse oximeter can be applied at various sites such as the finger and forehead, pulse oximetry at the sternum with the Electronic Patch is currently under investigation. In this paper the core technology is presented and future work will address the issue of using the technology.

\section{B. Packaging}

If the Electronic Patch is to be manufactured in large quantities, it would be preferable to manufacture the plastic parts by injection molding or by insert molding of the electronics and sensors. Possible materials must be biocompatible, have low attenuation of $2.4 \mathrm{GHz}$ radio signals, and very low absorption of water to protect the electronics and limit attenuation of 
TABLE IV

THE POWER CONSUMPTION OF THE ELECTRONIC PATCH COMPARED TO NEWER MiCROELECTRONIC CIRCUITS

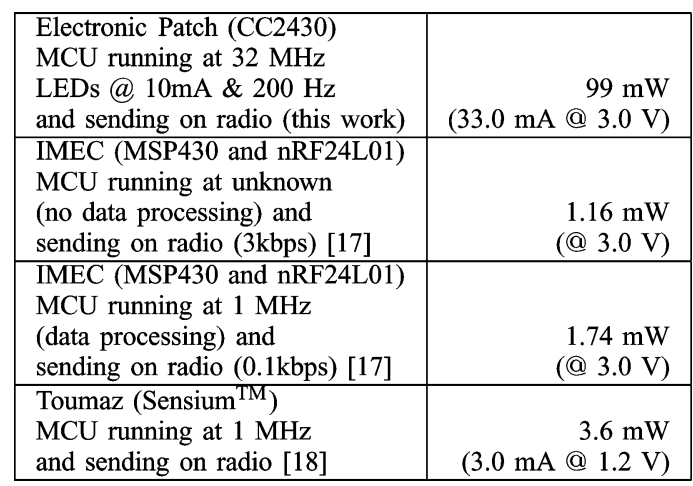

2.4 GHz radio signals by water. Promising materials are polylaurinlactam (PA12 or Nylon) and polyethylen (PEHD 300). Both materials are biocompatible. The maximum water uptake of PA12 is $12.8 \pm 4 \mathrm{~kg} / \mathrm{m}^{3}$ and for PEHD 300 it is less than $40 \mathrm{~g} / \mathrm{m}^{3}$ [29]. PEHD 300 is favorable because of its low attenuation of radio signals and very low water uptake.

\section{Power Consumption}

The power consumption of the Electronic Patch is currently too high for continuous wireless use with a coin size battery. Table IV shows the power consumption of the Electronic Patch compared to the power consumptions of reported microelectronic circuits and systems for wearable medical devices. The primary reason for a higher power consumption is that development of ultra low-power microelectronic components has not been the scope of this work and that the commercial components available at the time this work was initiated (primo 2007) had a higher power consumption compared to microelectronic components developed in the research environment. However, it was evident at the time that ultra low-power components would become commercial available. With the current electronic design, the following improvements could be done to decrease the power consumption: Duty cycle the SoC by employing the idle mode and lower the duty cycle of the LEDs. In Section V we outline how the power consumption could be reduced.

1) Reduction in Overall Power Consumption: The field of microelectronic circuits and SoCs for wearable medical electronics is currently evolving rapidly with a high number of scientific papers each year. This progress is also reflected in commercial technology, e.g., the introduction of the Sensium chip (Toumaz Technologies Limited). This chip can measure ECGs, process data at $1 \mathrm{MHz}$, and radio transmit data with a total power consumption of $3.6 \mathrm{~mW}$ [17]. Such systems could be employed in a new version of the Electronic Patch.

2) Local Versus Remote Data Computation: One possible way to reduce the power consumption is to reduce the SoC's workload by transmitting raw data to a remote server. The advantage using this model depends on the ratio between the radio power consumption, the power consumption of the SoC during computation, and the amount of data calculations. Yazicioglu $e t$ $a l$. [17] have demonstrated a system based on a MSP430 (Texas
Instruments) micro controller and a nRF24L01 (Nordic Semiconductor) radio. This system can record and transmit data at $3 \mathrm{kbps}$ using $0.77 \mathrm{~mW}$, proving that radio transmission is not necessarily a limiting factor.

Data in the Electronic Patch is represented with an average payload of 47 bits pr. measurement point. If this should be transmitted at $3 \mathrm{kbps}$ it would require a sampling frequency lower than $64 \mathrm{~Hz}$. Masimo Inc. uses, after downsampling, a $62.5 \mathrm{~Hz}$ signal in their algorithm [30] and a filter with a range of $0.57-4.2 \mathrm{~Hz}$. In comparison we use a filter range of $0.8-3 \mathrm{~Hz}$. It should therefore be possible to represent the data necessary to calculate the $\mathrm{SpO}_{2}$ and pulse rate with sampling frequency less than $64 \mathrm{~Hz}$.

3) Reduction in LED Power Usage: The current analog-todigital sample and conversion time is $163 \mu$ s and the LED "on" time is $560 \mu \mathrm{s}$. To handle noise by external light sources like florescent tubes with an oscillation frequency of twice the alternating power supply (i.e., $120 \mathrm{~Hz}$ in North America for $60 \mathrm{~Hz}$ power supply) a sampling frequency larger than $240 \mathrm{~Hz}$ should be used to avoid aliasing. ${ }^{3}$ E.g., a sampling frequency of $250 \mathrm{~Hz}$ will result in a duty cycle of $30 \%$ for the two LEDs. With a LED current of $10 \mathrm{~mA}$ at $1.5 \mathrm{~V}$ the average power consumption of two LEDs will be $4.5 \mathrm{~mW}$. A reduction in the power consumption by a factor of 5-10 is necessary for a true low power wireless sensor to be realized. The most obvious way to accommodate this is to reduce the LED "on" time and the ADC sample time. Another possible way is to utilize an architecture of integrate-and-hold instead of sample-and-hold; this approach also reduces the noise [24].

\section{CONCLUSION}

We have described the development and the individual components of the Electronic Patch in detail. The Electronic Patch is a new wearable health monitoring device. We believe this type of health monitoring by small non-invasive devices will become a new paradigm in health monitoring. We have demonstrated the Electronic Patch with reflectance pulse oximetry as this method provides valuable physiological information about both the heart and the lung function. The pulse oximetry solution in the Electronic Patch is based on a recently developed reflectance pulse oximetry sensor to lower the power consumption of the light sources. In this paper we have described the electronics and firmware embedded into the Electronic Patch. We have discussed and illustrated how the power consumption of the Electronic Patch could be lowered by using a newer generation of microelectronic systems for wearable medical electronics and optimizing the firmware and analog-to-digital conversion.

A physiologic evaluation of the Electronic Patch has been performed in a clinical setting in a self-testing study with three subjects. It is demonstrated that the Electronic Patch works as a

\footnotetext{
${ }^{3}$ It is not possible to lower the blinking frequency of the LEDs by applying a low-pass anti-aliasing filter to the photocurrent to filter out noise from background light oscillations. Since, if a low-pass filter is applied to the photocurrent it will be impossible to tell if the measured photocurrent is due to light originating from the LEDs or the background light oscillations. It is therefore a requirement that the LEDs blinking frequency is at least twice that of the oscillation frequency of other light sources.
} 
pulse oximeter. Larger scale clinical tests and trials of the Electronic Patch still remain to be undertaken including demonstration of the telemedicine capabilities of the device.

\section{ACKNOWLEDGMENT}

The authors would like to thank their collaborators within the Danish Innovation Consortium "The Electronic Patch." Especially, they would like to thank DELTA for a very fruitful collaboration. In particular, they would like to thank: M. Flintrup; K. Bach; I. Mangwiza; M. Otte and J. Janting from DELTA; and B. Kjeldgaard Vistisen from Coloplast A/S.

\section{REFERENCES}

[1] N. J. Holter and J. A. Generelli, "Remote recording of physiological data by radio," Rocky Mtn. Med. J., vol. 46, no. 9, pp. 747-751, Sep. 1949.

[2] S. R. Levine and M. Gorman, "Telestroke: The application of telemedicine for stroke," Stroke, vol. 30, no. 2, pp. 464-469, Feb. 1999.

[3] O. Wu and P. Langhorne, "The challenge of acute-stroke management: Does telemedicine offer a solution?," Int. J. Stroke, vol. 1, no. 4, pp. 201-207, Nov. 2006

[4] S. Scalvini, E. Zanelli, M. Volterrani, G. Martinelli, D. Baratti, O. Buscaya, P. Baiardi, F. Glisenti, and A. Giordano, "A pilot study of nurseled, home-based telecardiology for patients with chronic heart failure," J. Telemed. Telecare, vol. 10, no. 2, pp. 113-117, 2004.

[5] S. Scalvini, S. Capomolla, E. Zanelli, M. Benigno, D. Domenighini, L. Paletta, F. Glisenti, and A. Giordano, "Effect of home-based telecardiology on chronic heart failure: Costs and outcomes," J. Telemed. Telecare, vol. 11 (Suppl. 1), pp. 16-18, 2005.

[6] B. Dinesen, C. Nøhr, S. K. Andersen, H. Sejersen, and E. Toft, "Under surveillance, yet looked after: Telehomecare as viewed by patients and their spouse/partners," Eur. J. Cardiovasc. Nurs., vol. 7, no. 3, pp. 239-246, Sep. 2008.

[7] J. M. Kang, T. W. Yoo, and H. C. Kim, "A wrist-worn integrated health monitoring instrument with a tele-reporting device for telemedicine and telecare," IEEE Trans. Instrum. Meas., vol. 55, no. 5, pp. 1655-1661, 2006.

[8] U. Anliker, J. A. Ward, P. Lukowicz, G. Troster, F. Dolveck, M. Baer, F. Keita, E. B. Schenker, F. Catarsi, L. Coluccini, A. Belardinelli, D. Shklarski, M. Alon, E. Hirt, R. Schmid, and M. Vuskovic, "Amon: A wearable multiparameter medical monitoring and alert system," IEEE Trans. Inf. Technol. Biomed., vol. 8, no. 4, pp. 415-427, 2004.

[9] Y. Mendelson, R. J. Duckworth, and G. Comtois, "A wearable reflectance pulse oximeter for remote physiological monitoring," in Proc. IEEE Engineering in Medicine and Biology Society Conf., 2006, vol. 1, pp. 912-915 [Online]. Available: http://dx.doi.org/10.1109/IEMBS. 2006.260137

[10] S. Park, C. Gopalsamy, R. Rajamanickam, and S. Jayaraman, "The wearable motherboard: A flexible information infrastructure or sensate liner for medical applications," Stud. Health Technol. Inform., vol. 62, pp. 252-258, 1999.

[11] R. Paradiso, G. Loriga, and N. Taccini, "A wearable health care system based on knitted integrated sensors," IEEE Trans Inf. Technol. Biomed., vol. 9, no. 3, pp. 337-344, Sep. 2005.

[12] R. Paradiso, A. Alonso, D. Cianflone, A. Milsis, T. Vavouras, and C. Malliopoulos, "Remote health monitoring with wearable non-invasive mobile system: The healthwear project," in Proc. IEEE Engineering in Medicine and Biology Society Conf., 2008, vol. 2008, pp. 1699-1702 [Online]. Available: http://dx.doi.org/10.1109/IEMBS.2008.4649503

[13] R. G. Haahr, S. Duun, K. Birkelund, P. Raahauge, P. Petersen, H. Dam, L. Nørgaard, and E. V. Thomsen, "A novel photodiode for reflectance pulse oximetry in low-power applications," in Proc. Ann. Int. Conf. IEEE Engineering in Medicine and Biology Society, 2007, vol. 2007, pp. 2350-2353 [Online]. Available: http://dx.doi.org/10.1109/IEMBS. 2007.4352798

[14] S. Duun, R. G. Haahr, K. Birkelund, P. Raahauge, P. Petersen, H. Dam, L. Noergaard, and E. V. Thomsen, "A novel ring shaped photodiode for reflectance pulse oximetry in wireless applications," in Proc. IEEE Sensors, 2007, pp. 596-599.

[15] S. B. Duun, R. G. Haahr, K. Birkelund, and E. V. Thomsen, "A ringshaped photodiode designed for use in a reflectance pulse oximetry sensor in wireless health monitoring applications," IEEE Sensors J., vol. 10, no. 2, pp. 261-268, 2010.
[16] R. G. Haahr, S. Duun, E. V. Thomsen, K. Hoppe, and J. Branebjerg, "A wearable electronic patch for wireless continuous monitoring of chronically diseased patients," in Proc. 5th Int. Summer School and Symp. Medical Devices and Biosensors, 2008, pp. 66-70.

[17] R. Yazicioglu, T. Torfs, J. Penders, I. Romero, H. Kim, P. Merken, B. Gyselinckx, H. Yoo, and C. Van Hoof, "Ultra-low-power wearable biopotential sensor nodes," in Proc. IEEE Annu. Int. Conf. Engineering in Medicine and Biology Society, Sep. 3-6, 2009, pp. 3205-3208.

[18] A. C. W. Wong, D. McDonagh, O. Omeni, C. Nunn, M. Hernandez-Silveira, and A. J. Burdett, "Sensium: An ultra-low-power wireless body sensor network platform: Design \& application challenges," in Proc. IEEE Annu. Int. Conf. IEEE Engineering in Medicine and Biology Society, 2009, pp. 6576-6579.

[19] M. Tavakoli, L. Turicchia, and R. Sarpeshkar, "An ultra-low-power pulse oximeter implemented with an energy-efficient transimpedance amplifier," IEEE Trans. Biomed. Circuits Syst., vol. 4, no. 1, pp. 27-38, 2010.

[20] T. Aoyagi, M. Kishi, K. Yamaguchi, and S. Watanabe, "Improvement of the earpiece oximeter," in Proc. 13th Annu. Meeting Japanese Societry of Medical Electronics and Biological Engineering Abstracts, 1974, pp. 90-91.

[21] Y. Mendelson, P. W. Cheung, M. R. Neuman, D. G. Fleming, and S. D. Cahn, "Spectrophotometric investigation of pulsatile blood flow for transcutaneous reflectance oximetry," Adv. Exp. Med. Biol., vol. 159, pp. 93-102, 1983.

[22] Y. Mendelson, J. C. Kent, B. L. Yocum, and M. J. Birle, "Design and evaluation of a new reflectance pulse oximeter sensor," Med. Instrum., vol. 22, no. 4, pp. 167-173, Aug. 1988.

[23] Y. Mendelson and C. Pujary, "Measurement site and photodetector size considerations in optimizing power consumption of a wearable reflectance pulse oximeter," in Proc. 25th IEEE Annu. Int. Conf. Engineering in Medicine and Biology Society, 2003, vol. 4, pp. 3016-3019.

[24] S. Duun, R. G. Haahr, O. Hansen, K. Birkelund, and E. V. Thomsen, "High quantum efficiency annular backside silicon photodiodes for reflectance pulse oximetry in wearable wireless body sensors," J. Micromech. Microeng., vol. 20, no. 7, pp. 075020-075020, 2010.

[25] P. Samuelsen, "Dressing with hydrocolloid," U.S. Patent 4867 748, 1987.

[26] H. Ding, J. Q. Lu, W. A. Wooden, P. J. Kragel, and X.-H. Hu, "Refractive indices of human skin tissues at eight wavelengths and estimated dispersion relations between 300 and 1600 nm," Phys. Med. Biol., vol. 51, no. 6, pp. 1479-1489, Mar. 2006.

[27] T. Jensen, S. Duun, J. Larsen, R. G. Haahr, M. H. Toft, B. Belhage, and E. V. Thomsen, "Independent component analysis applied to pulse oximetry in the estimation of the arterial oxygen saturation ( $\mathrm{SpO} 2)-\mathrm{A}$ comparative study," in Proc. IEEE Annu. Int. Conf. Engineering in Medicine and Biology Society, 2009, pp. 4039-4044.

[28] J. G. Webster, S. A. Clark, D. J. Sebald, J. Farmer, O. Wieben, B. W. J. Bourgeois, J. S. Schowalter, M. V. S. Reddy, K. S. Paranjape, S. Tungjitkusolmun, A. Lozano-Nieto, and J. B. Ruchala, Design of Pulse Oximeters, J. G. Webster, Ed. New York: Taylor \& Francis, 1997.

[29] J. Janting, "Water penetration study for a 3d electronic patch," in Proc. IMAPS Nordic Annu. Conf., Helsingør, Denmark, Sep. 14-16, 2008, pp. 101-108.

[30] M. K. Diab, E. Kinai-Azarbayjany, I. M. Elfadel, R. J. McCharthy, W. M. Weber, and R. A. Smith, "Signal processing apperatus," U.S. Patent 7383070 B2, 2008.

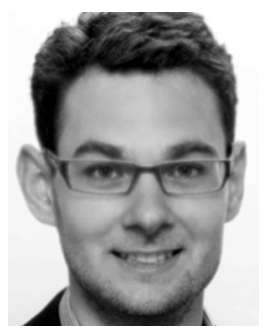

Rasmus G. Haahr (M'11) was born in Copenhagen, Denmark in 1982. He received the M.Sc. degree in engineering physics and the Ph.D. degree from the Technical University of Denmark (DTU), Lyngby, in 2006 and 2010, respectively.

Currently, he is in the Research and Development Division, DELTA Microelectronics, Hørsholm, Denmark, developing biomedical sensors and signal processing technologies for the Electronic Patch. From 2008 to 2009, he joined the Massachusetts Institute of Technology as a Visiting Researcher working on an implantable behavior monitoring system for small animals in collaboration with Massachusetts General Hospital. Since 2007, he has been with the MEMS Applied Sensors Group, Department of Micro and Nanotechnology, DTU, working on the development of a wearable physiological monitoring system-the Electronic Patch. 


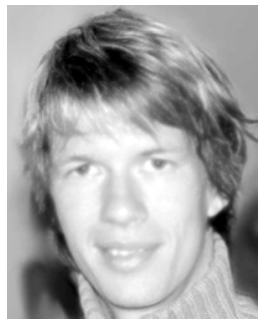

Sune B. Duun was born in Denmark in 1977. He received the M.Sc. degree and the Ph.D. degree from the Technical University of Denmark (DTU), Lyngby, in 2006 and 2010, respectively.

Currently, he is in the Research and Development Division, Topsil Semiconductor Materials A/S, Frederikssund, Denmark. From 1997 to 1999, he did military service in the army and currently holds a rank of First Lieutenant. From 2001 to 2005, he worked part-time at BBT Fiberoptic, Denmark, as Sales Engineer of Avantes fiber-optic spectrometers. In 2006, he joined the Department of Micro and Nanotechnology, DTU, working on the development of pulse oximetry sensors for the wearable monitoring system-the Electronic Patch.

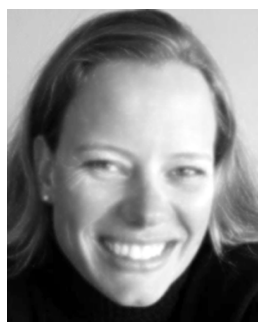

Mette H. Toft was born in Copenhagen, Denmark, in 1977. She graduated as M.D. from the University of Copenhagen, Copenhagen, Denmark, in 2005.

After graduation, she has worked at the Department of Clinical Physiology, Copenhagen University Hospital, Bispebjerg, and taught respiratory and cardiovascular physiology part-time at the Medical Faculty at the University of Copenhagen. She has worked in Greenland for seven months and the past two years she has focused on anaesthesiology. Currently, she is doing her residency at Copenhagen University Hospital, Herlev, specializing in anaesthesiology.

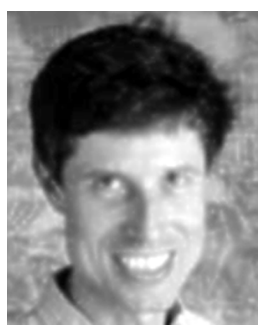

Bo Belhage was born in Copenhagen, Denmark, in 1962. He graduated as M.D. from the Medical School, University of Copenhagen, Copenhagen, Denmark, in 1988. He received the DMSc degree in 1996.

He became a specialist in anaesthesiology and a Clinical Associate Professor in 2005. In 2007, he became Chief Anesthesiologist for abdominal surgery at Bispebjerg Hospital, Copenhagen, Denmark. He has taught pre- and postgraduate students, as well as Ph.D. students in biochemistry and physiology since 1994. He is an Examiner at Odense University, Odense, Denmark, and Aarhus University, Herning, Denmark.

Dr. Belhage has authored more than 50 biomedical papers and book chapters and serves as reviewer for several journals.

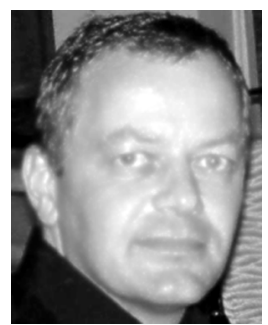

Jan Larsen (SM'03) received the M.Sc. and Ph.D. degrees in electrical engineering from the Technical University of Denmark (DTU), Lyngby, in 1989 and 1994, respectively.

Currently, he is an Associate Professor in the Cognitive Systems Section at the at Department of Informatics and Mathematical Modelling, DTU, and Director of the Danish Sound Technology Network. He has authored and coauthored more than 100 papers and book chapters within the areas of nonlinear statistical signal processing, machine learning, neural net- works and datamining with applications to biomedicine, monitoring systems, multimedia, and webmining. He has participated in several national and international research programs, and has served as a reviewer for many international journals, conferences, publishing companies, and research funding organizations.

Dr. Larsen has taken part in conference organizations, including the IEEE Workshop on Machine Learning for Signal Processing (formerly Neural Networks for Signal Processing) 1999-2010. He is a former Chair of the IEEE Machine Learning for Signal Processing Technical Committee of the IEEE Signal Processing Society (2005-2007), and Chair of IEEE Denmark Section's Signal Processing Chapter (2002-present). Other professional committee participation includes: Member of the Technical Committee 14: Signal Analysis for Machine Intelligence of the International Association for Pattern Recognition since 2006; Steering Committee Member of the Audio Signal Processing Network in Denmark since 2006; Editorial Board Member of Signal Processing, Elsevier, 2006-2007; and guest editorships involving IEEE TRANSACTIONS ON NEURAL NETWORKS; Journal of VLSI Signal Processing Systems; and Neurocomputing.

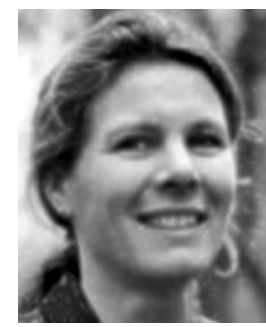

Karen Birkelund was born in Køge, Denmark, in 1966. She received the M.Sc. degree from Roskilde University Center, Roskilde, Denmark, in 1993 and the $\mathrm{Ph} . \mathrm{D}$. degree in electrical engineering from the Technical University of Denmark (DTU), Lyngby, in 1997.

From 1998 to 2002, she was at Danfoss A/S as a Research Engineer within MEMS pressure sensors. From 2002 to 2004, she was at Cantion A/S as a Research Engineer within cantilever-based sensor systems. From 2004 to 2006, she was with TopSpin $\mathrm{A} / \mathrm{S}$ also as a Research Engineer within silicon-based dispensing systems for biotechnology research. In 2006, she joined the Department of Micro and Nanotechnolgy, DTU, as a Postdoctoral working mainly on MEMS devices and encapsulation for harsh environments.

Dr. Birkelund has exceptional experience in spin-off companies and commercialization of micro and nanotechnology. She is currently pursuing a tenure track academic career.

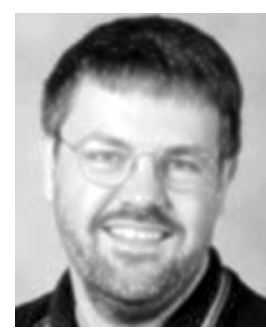

Erik V. Thomsen was born in Aarhus in 1964. He received the M.Sc. degree in physics from Odense University, Odense, Denmark, and the Ph.D. degree in electrical engineering from the Technical University of Denmark (DTU), Lyngby, in 1998.

He has been affiliated with the Department for Micro and Nanotechnology, DTU, since 1992. In 2008, he became a Professor. Since 2003, he has been group leader for the MEMS Applied Sensors Group. His current research and teaching interests include MEMS multisensors, piezo resistivity of strained layers, piezoelectric MEMS devices, and microsystem packaging. He teaches classes in solid-state electronics, microtechnology, and nano- and microfabrication.

Dr. Thomsen received the prestigious AEG Electron Prize in 1995 and has also received numerous teaching awards at DTU. 